\title{
Identification of novel snRNA-specific Sm proteins that bind selectively to U2 and U4 snRNAs in Trypanosoma brucei
}

\author{
ITAI DOV TKACZ, ${ }^{1}$ YANIV LUSTIG, ${ }^{1}$ MICHAEL ZEEV STERN, ${ }^{1}$ MOSHE BITON, ${ }^{1}$ MALI SALMON-DIVON, ${ }^{1}$ \\ ANISH DAS, ${ }^{2}$ VIVIAN BELLOFATTO, ${ }^{2}$ and SHULAMIT MICHAELI ${ }^{1}$ \\ ${ }^{1}$ The Mina \& Everard Goodman Faculty of Life Sciences, Bar-Ilan University, Ramat-Gan 52900, Israel \\ ${ }^{2}$ Department of Microbiology and Molecular Genetics, UMDNJ-New Jersey Medical School, International Center for Public Health, \\ Newark, New Jersey 07103, USA
}

\begin{abstract}
In eukaryotes the seven Sm core proteins bind to U1, U2, U4, and U5 snRNAs. In Trypanosoma brucei, Sm proteins have been implicated in binding both spliced leader (SL) and U snRNAs. In this study, we examined the function of these Sm proteins using RNAi silencing and protein purification. RNAi silencing of each of the seven Sm genes resulted in accumulation of SL RNA as well as reduction of several $U$ snRNAs. Interestingly, U2 was unaffected by the loss of SmB, and both U2 and U4 snRNAs were unaffected by the loss of SmD3, suggesting that these snRNAs are not bound by the heptameric Sm complex that binds to U1, U5, and SL RNA. RNAi silencing and protein purification showed that U2 and U4 snRNAs were bound by a unique set of Sm proteins that we termed SSm (specific spliceosomal Sm proteins). This is the first study that identifies specific core Sm proteins that bind only to a subset of spliceosomal snRNAs.
\end{abstract}

Keywords: trypanosome; snRNA; Sm proteins; SL RNA; Lsm

\section{INTRODUCTION}

Sm proteins are core proteins in mammals and yeast that bind the spliceosomal U1, U2, U4, and U5 snRNAs (Will and Lührmann 2001). The Sm proteins are characterized by bipartite sequence motifs termed Sm1 and Sm2 (Hermann et al. 1995; Séraphin 1995) that function in protein-protein interactions (Hermann et al. 1995). The Sm proteins bind to the Sm-site, which consists of a single-stranded uridinerich region that is usually flanked by two stem-loop structures (Branlant et al. 1982). The seven Sm proteins SmB, D1, D2, D3, E, F, and G bind to the Sm-site, forming the Sm core (Branlant et al. 1982; Liautard et al. 1982). In the absence of $U$ snRNA, three stable complexes of Sm proteins, D3/B, D1/D2, and E/F/G, are found (Hermann et al. 1995; Raker et al. 1996). None of the individual Sm proteins binds stably to RNA; instead, the Sm proteins interact via their Sm 1 and 2 motifs. The RNA binding site is generated as a consequence of Sm heteromeric formation

Reprint requests to: Shulamit Michaeli, The Mina \& Everard Goodman Faculty of Life Sciences, Bar-Ilan University, Geha Street, Ramat-Gan 52900, Israel; e-mail: michaes@mail.biu.ac.il; fax: 972-3-5351824.

Article published online ahead of print. Article and publication date are at http://www.rnajournal.org/cgi/doi/10.1261/rna.174307.
(Raker et al. 1996). The crystal structures of two Sm protein complexes, D3/B and D1/D2, show that these proteins have a common fold containing an N-terminal helix, followed by a five-stranded antiparallel $\beta$-sheet (Kambach et al. 1999).

In addition to the core Sm proteins, other proteins carrying Sm motifs have been identified in many eukaryotes. These proteins are designated Lsm (Like-Sm) proteins. Two distinct complexes containing these proteins exist. One complex, composed of Lsm2p-Lsm8p, binds the $3^{\prime}$-end poly $(\mathrm{U})$ tract of U6 snRNA, and is required for U6 snRNP formation (Terns et al. 1992; Achsel et al. 1999). The binding of Lsm2p-Lsm8p to the 3' U-rich single-stranded region of U6 facilitates U6 RNP formation and assembly of this snRNA with U4 into the di-snRNP and probably the tri-snRNP complexes (Achsel et al. 1999; Mayes et al. 1999; Salgado-Garrido et al. 1999). The second complex, consisting of Lsm1p-Lsm7p, functions in mRNA decay in the cytoplasm, mostly during the decapping step (Bouveret et al. 2000). Additional Lsm proteins exist; these include those that form the U7 snRNP, which functions in $3^{\prime}$-end processing of histone mRNA. In this case, the canonical D1 and D2 Sm proteins are replaced by Lsm10 and Lsm11 (Pillai et al. 2001, 2003).

Trypanosomes are protozoan parasites that diverged very early in the eukaryotic lineage (Stevens and Gibson 1999). 
These parasites possess very exotic RNA processing mechanisms such as trans-splicing and RNA editing (Liang et al. 2003; Simpson et al. 2003). Trypanosomes possess the full set of $U$ snRNAs that function in splicing (Liang et al. 2003). These RNAs, as opposed to U snRNAs in other eukaryotes, carry various cap structures. U1 (Schnare and Gray 1999; Djikeng et al. 2001; Palfi et al. 2005), U2, and U4 possess a tri-methyl guanosine cap (Mottram et al. 1989). U5 has a 5' phosphate end (Dungan et al. 1996; $\mathrm{Xu}$ et al. 1997; Bell and Bindereif 1999). The U6 cap is a $\gamma$-methyl cap that is identical to the cap found in other eukaryotes (Mottram et al. 1989). The spliced leader (SL) RNA has a unique cap that is composed of $m^{7} G$ together with four hypermodified nucleotides, which comprises a cap-4 (Bangs et al. 1992). Like U snRNAs in other eukaryotes, trypanosome snRNAs carry Sm-sites, but this site is very degenerate in the U2 and U4 snRNAs (Mottram et al. 1989).

The first trypanosome Sm protein was identified as an SL RNA core protein (Goncharov et al. 1999), and later the full set of seven Sm proteins was identified (Palfi et al. 2000). The trypanosome Sm proteins carry bipartite Sm motifs including interesting deviations from the $\mathrm{Sm}$ consensus (Goncharov et al. 1999; Palfi et al. 2000). The C terminus of the $\mathrm{Sm}$ proteins lacks the RG dipeptide repeats in which the arginine of this motif is methylated in the mammalian proteins. This finding may explain in part why trypanosome Sm proteins are not recognized by anti-Sm antibodies (Palfi et al. 2000). To elucidate the repertoire and function of Sm proteins, two Sm proteins, SmE and SmD1, were silenced by RNAi (Mandelboim et al. 2003). As expected, the silencing of these proteins decreased the level of snRNAs, but surprisingly, the level of SL RNA increased more than 10-fold. The SL RNA that accumulates during silencing lacks the +4 cap nucleotide and forms a stable RNP complex called SL RNP-C (Mandelboim et al. 2003). Lsm proteins were also identified in Trypanosoma brucei (Liu et al. 2004). Two of these proteins (homologs to Lsm3p and Lsm8p) were found to be essential for U6 stability and mRNA decay (Liu et al. 2004).

Relatively little is known about snRNP biogenesis in trypanosomes. Specifically, it is not clear in which cellular compartment this process takes place. The U snRNAs are transcribed in trypanosomes by RNA polymerase III from extragenic tRNA promoters (Nakaar et al. 1994). In contrast, the SL RNA is transcribed by an RNA polymerase II-dependent extragenic promoter (Gilinger and Bellofatto 2001; Das et al. 2005). Strong evidence was provided for coupling of the unique hypermethylated capping of SL RNA and its transcription (Mair et al. 2000). Most recently two distinct methyltransferases that carry out the $2^{\prime}-O^{\prime}$ methylations at positions +3 and +4 and +2 of the cap- 4 were identified and localized to the nucleus (Arhin et al. 2006a,b) . Despite the fact that many factors involved in SL RNA biogenesis are found within the nucleus, studies from recent years suggested that SL RNA biogenesis, neverthe- less, involves a cytoplasmic phase (Mandelboim et al. 2003; Zeiner et al. 2003). It was suggested that export of SL RNA to the cytoplasm is mediated by XPO1 (Zeiner et al. 2003). The finding that SL RNA accumulated in the cytoplasm during Sm depletion, and that snRNP assembly occurs in the cytoplasm in metazoa, led to the proposal that SL RNA assembly with Sm proteins takes place in the cytoplasm in trypanosomes (Mandelboim et al. 2003). Most recently, inspecting the localization of SL RNA during early time points of Sm depletion indicated that SL RNA first accumulates in the nucleus. Only after massive accumulation, the SL RNA migrates to the cytoplasm, forming speckles as previously described. RNAi of XPO1 also demonstrates that this factor does not function in SL RNA transport (Biton et al. 2006). These recent data suggest that SL RNA assembly with $\mathrm{Sm}$ proteins most probably takes place in the nucleus.

In this study, we examined the function of all seven Sm proteins in trypanosomes and three additional proteins carrying Sm domains. Surprisingly, the two proteins similar to known Lsm proteins (Liu et al. 2004) were found to be unusual Sm proteins that bind selectively to either U2 or U4 snRNPs. We termed these proteins SSm (specific spliceosomal Sm proteins), to distinguish them from previously characterized Sm and Lsm proteins. Using RNAi silencing and affinity-tagging of Sm and SSm proteins, we determined the selectivity of their binding to SL and snRNAs. This is the first study to describe U spliceosomal snRNPs (U2, U4) that have unique core Sm proteins. This finding contributes to our understanding how trypanosomes selectively distinguish and control the assembly of core proteins on snRNAs, which function in splicing but harbor different cap structures.

\section{RESULTS}

\section{Silencing of $\mathrm{Sm}$ core proteins results in an increase in the steady-state level of undermethylated SL RNA and differential decrease in $U$ snRNAs}

In our previous studies, silencing of either SmE or SmD1 in T. brucei revealed a very characteristic phenotype: U snRNA levels were reduced, although SL RNA levels increased 10-fold (Mandelboim et al. 2003). To examine whether alterations in other Sm proteins generated this phenotype, additional Sm proteins were individually down-regulated by RNAi. In the silencing constructs, T7 promoters were used to produce the gene-specific dsRNA under tetracycline regulation (Wang et al. 2000). Parasites stopped growing within $3 \mathrm{~d}$ after dsRNA induction in each case (data not shown). RNA was prepared from uninduced cells and from cells after $2 \mathrm{~d}$ of induction, and analyzed using snRNAspecific probes (Fig. 1A). Quantification of data by densitometry from three independent experiments is shown in Figure 1B. In each case, the reduction of the specific Sm protein resulted in an increase in SL RNA amounts and 
A
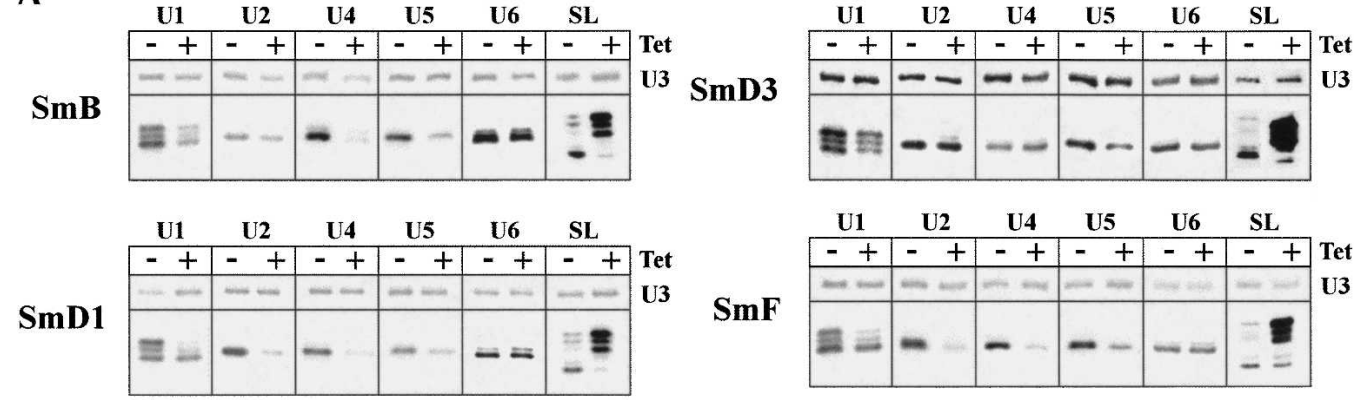

SmF
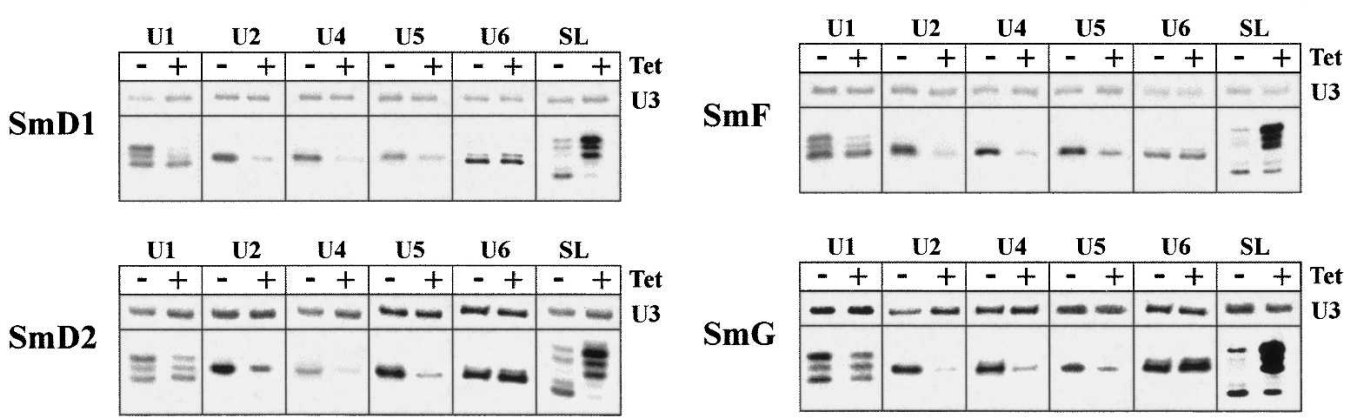

B
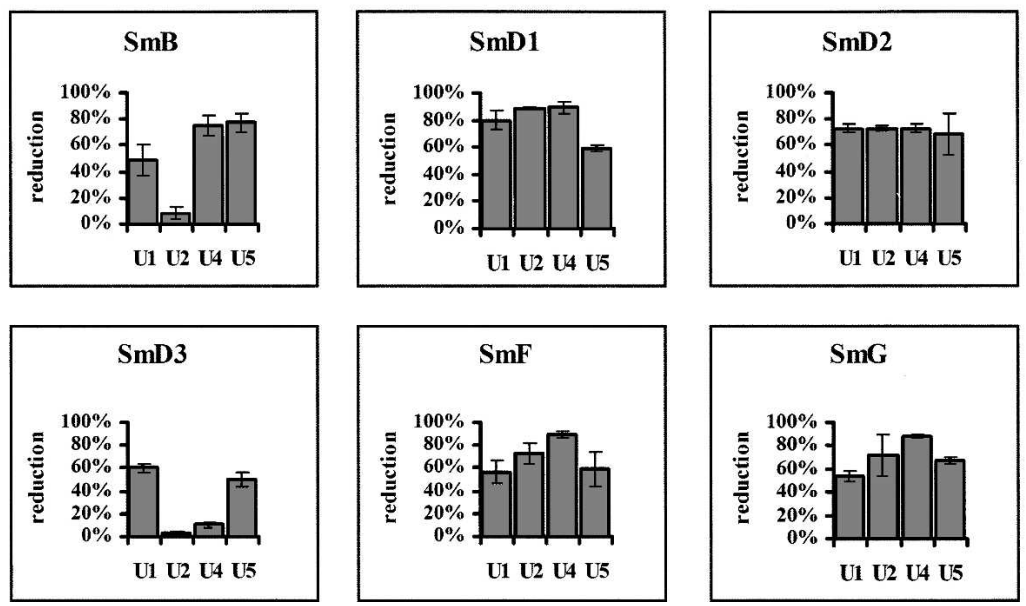

FIGURE 1. Effect of Sm proteins silencing on the level of snRNAs. (A) Total RNA was prepared from cells carrying the SmB, SmD1, SmD2, SmD3, SmF, and SmG RNAi constructs before (-Tet) and after $3 \mathrm{~d}$ of induction with tetracycline (+Tet), essentially as described (Mandelboim et al. 2003). Total RNA (10 $\mu \mathrm{g})$ was subjected to primer extension with radiolabeled oligonucleotides complementary to U1, U2, U3, U4, U5, U6, and SL RNA. The oligonucleotides and their sequences are summarized in Materials and Methods. The extension products were separated on $6 \%$ denaturing gels. (B) Quantitative analysis shows the percent reduction in the level of snRNAs, as determined by densitometry of three independent experiments. Standard deviation is indicated by error bars. The level of U3 snoRNA was used to normalize the level of RNA in each sample.

a distinct decrease in U1, U2, U4, and U5 snRNA levels. As was previously observed, SL RNA that accumulated during Sm silencing lacked the +4 cap modification (Mandelboim et al. 2003), suggesting that this modification event is linked to assembly with Sm proteins. Reduction levels for each U snRNA were distinct for each Sm protein. Overall, silencing of SmD1, D2, E, F, or G reduced the levels of U1, U2, U4, and U5 by 55\%-90\%. Similar results were published for SmE (Mandelboim et al. 2003). Silencing of $\mathrm{SmB}$ caused a reduction in U1, U4, and U5 snRNAs by $49 \% \pm 12 \%, 75 \% \pm 7.7 \%$, and $77 \% \pm 7 \%$, respectively. However, U2 snRNA levels were only marginally affected $(8.5 \% \pm 4.5 \%)$. Similarly, silencing of SmD3 resulted in reduction of $\mathrm{U} 1$ and $\mathrm{U} 5$ by $60 \% \pm 3.5 \%$ and $50 \% \pm 6 \%$, respectively, whereas levels of U2 were reduced by only
$3 \% \pm 2.1 \%$ and $\mathrm{U} 4$ snRNAs by $10 \% \pm 2 \%$. These results suggest that different combinations of Sm proteins, possibly in distinct Sm heptameric complexes, bind to the SL RNA and subsets of $U$ snRNAs in different ways.

\section{Affinity selection using TAP-tagged Sm proteins demonstrates differential association of snRNAs with $\mathrm{SmB}$ and SmD3 proteins}

To verify that the Sm proteins have differential affinity to $U$ snRNAs, fusion proteins containing a tandem affinity (TAP) tag were used to transfect procyclic T. brucei parasites, as described in Materials and Methods. The expression of the tagged proteins in clonal cell lines was confirmed by Western analysis (Fig. 2-A). All fusion protein constructs 


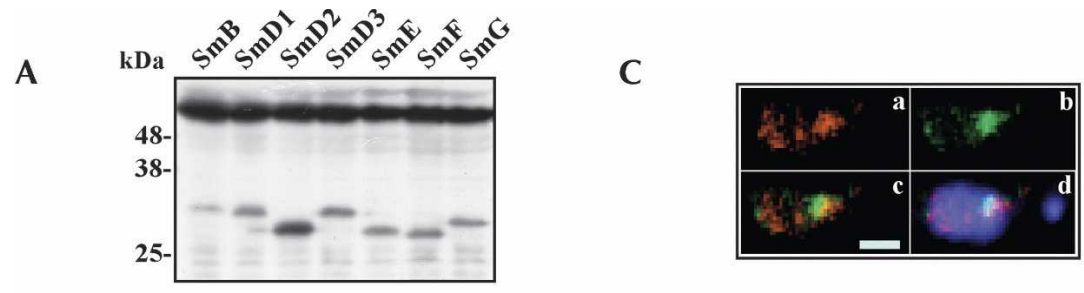

B

a

b

\begin{tabular}{|c|c|c|c|c|c|c|}
\hline & U3 & U1 & U2 & U4 & U5 & SL \\
\hline \multirow{2}{*}{$\mathrm{SmB}$} & $\begin{array}{lll}\text { T } & \mathbf{S} & \mathbf{B} \\
\end{array}$ & $\begin{array}{lll}\text { T } & \text { S } & \text { B } \\
\end{array}$ & T $\mathrm{S} \quad \mathrm{B}$ & T S S B & T S S B & $\begin{array}{lll} & S & B \\
\end{array}$ \\
\hline & - & 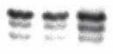 & $=-\cdots$ & $-\infty$ & $-\infty$ & $\mathrm{a}=\mathrm{E}$ \\
\hline
\end{tabular}

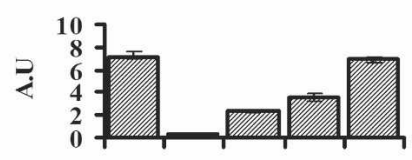

U1 U2 U4 U5 SL

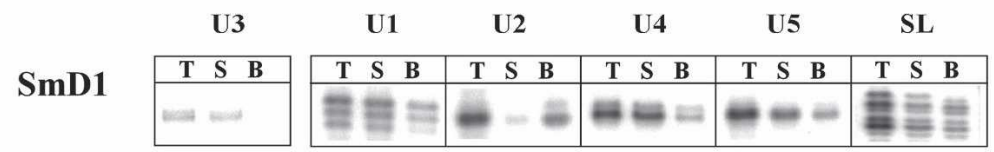

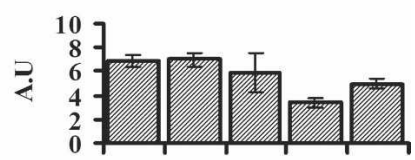

U1 U2 U4 U5 SL

\begin{tabular}{|c|c|c|c|c|c|c|}
\hline \multirow{3}{*}{ SmD2 } & U3 & U1 & U2 & U4 & U5 & SL \\
\hline & T S B & T S B & $\begin{array}{lll}\text { T } & \text { S } & \text { B } \\
\end{array}$ & $\begin{array}{lll}\text { T } & \text { S } & \text { B } \\
\end{array}$ & $\begin{array}{lll}\text { T } & \mathbf{S} & \mathbf{B} \\
\end{array}$ & 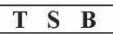 \\
\hline & $=\infty$ & L 2 를 & $--\mathbf{B}$ & $--=$ & $--\mathbf{0}$ & 를롤 \\
\hline
\end{tabular}

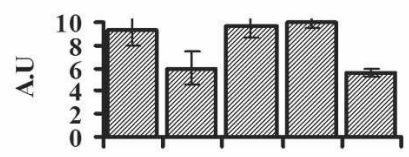

U1 U2 U4 U5 SL

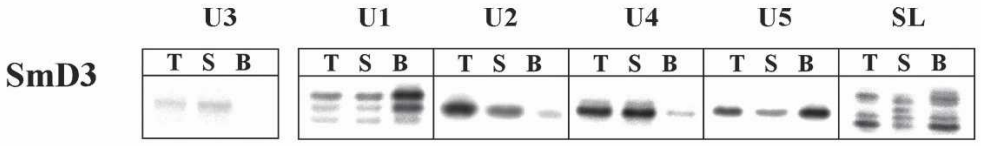

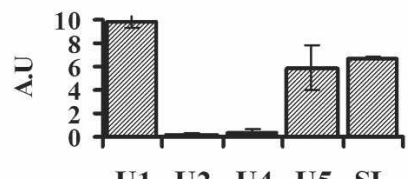

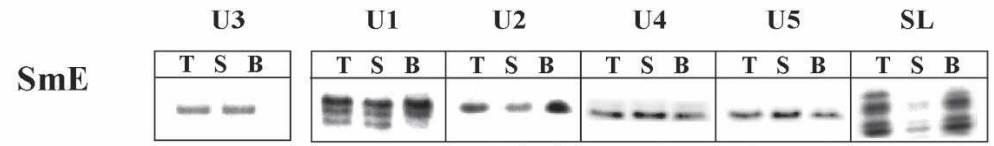

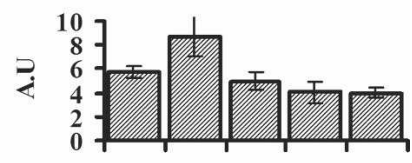

U1 U2 U4 U5 SL

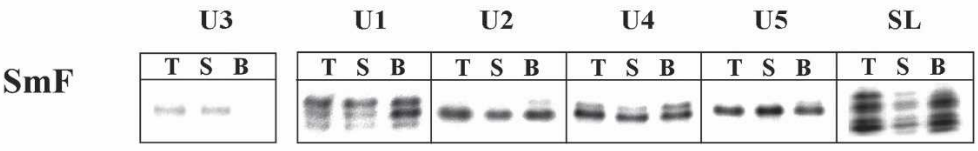

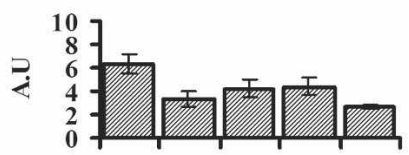

U1 U2 U4 U5 SL

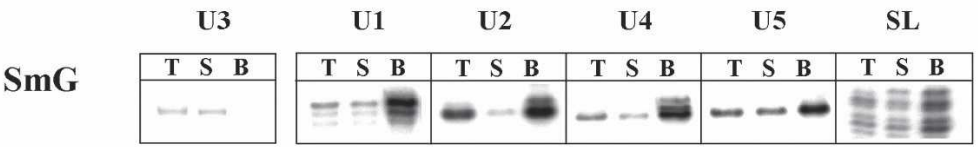

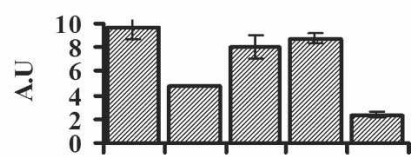

U1 U2 U4 U5 SL

FIGURE 2. (Legend on next page) 
produced polypeptides of the expected molecular mass. The expression level of the different tagged proteins was very similar, with the exception of SmD2, which was highly expressed. To determine which snRNAs are associated with specific Sm proteins, fusion proteins along with bound RNA were affinity-purified based on the tight association of the protein A, within the TAP tag, to IgG-conjugated beads. RNA was extracted from the bound material by phenol, and the specific snRNAs were identified by primer extension (Fig. 2B). The different efficiency of selection can be visualized by comparing the amount of RNA selected on the beads with an aliquot of the total amount of the snRNA present in the sample. The data enable us to examine the relative efficiency of selection of each of the snRNAs by the different proteins, as well as to compare the efficiency of selection of the snRNAs among the different Sm proteins. Great variation exists in the efficiency of affinity selection among the different tagged proteins. Quantitating the efficiency of selection of each snRNA from three independent experiments in arbitrary units is shown in Figure 2B-b. The tagged proteins SmD2, SmD3, and SmG selected the particles in the same efficiency ( $\sim 10$ arbitrary units), presenting $10 \%-15 \%$ of snRNPs present in the extract. Tagged SmD1, SmB, SmE, and SmF selected only 5\%-7\% of the snRNPs. Despite differences in the efficacy of selection, results demonstrate high specificity in the ability of the tagged proteins to select the different snRNAs. The results clearly show that $\mathrm{SmD} 1, \mathrm{D} 2, \mathrm{E}, \mathrm{F}$, and $\mathrm{G}$ are bound to U1, U2, U4, and U5 snRNAs. These protein-RNA interactions are specific, as U3 snoRNA was not detected in any of the Sm-protein-dependent purifications. The characteristic association of RNA and Sm proteins are consistent with the data shown in Figure 1, indicating that SmD1, D2, E, F, and G proteins are important for the assembly/ stabilization of the U1, U2, U4, U5, and SL RNAcontaining snRNPs. Conversely, both Sm B and D3 did not bind to U2 snRNA. The U2 snRNP was not selected by $\mathrm{SmB}$ in contrast to the selection of U1, U4, U5, and SL RNA. Moreover, the absence of these two proteins in the RNAi experiments did not lead to a decrease in U2 snRNA levels, as shown in Figure 1. SmD3 did not bind to $\mathrm{U} 2$ or $\mathrm{U} 4$ snRNAs, but did bind to $\mathrm{U} 1$ and U5 snRNAs. These results are in agreement with the effect of silencing the SmD3 by RNAi, as shown in Figure 1.

\section{Localization and assembly of Sm tagged proteins to their cognate snRNPs}

To investigate the source of variation leading to the difference in the efficiency of selection, several factors such as the level of expression, toxicity created by the expression of the tagged protein, and assembly of the tagged protein into snRNP complexes were examined. The effect of expression of the tagged proteins on cell viability and growth rate was examined in cells expressing two tagged Sm proteins, SmG and $\mathrm{SmD1}$, which select the snRNPs efficiently or poorly, respectively. The results indicate no difference in the growth rate upon induction of tagged-Sm protein synthesis, suggesting that expression of these proteins is not toxic to the cells (data not shown).

We next examined the localization of the tagged proteins. To this end, an SmE-GFP construct was prepared. The expression of the protein is constitutive and is driven by the EP promoter (explained in Materials and Methods). The results indicate that the SmE tagged protein is concentrated mainly in the nucleus in a large "dot," but also in smaller "dots" (like speckles) (see Fig. 2C-b). Hybridization with SL RNA suggests that the single "dot" contained the majority of the SL RNA (Fig. 2C-a). The merged images of the SmE-GFP and SL RNA without or with nuclear staining, respectively, are presented in Figure 2C, panels $c$ and $d$. Results suggest that the majority of the SL RNA bound to Sm proteins is confined to a distinct compartment within the nucleus.

The presence of a large TAP-tag of $23 \mathrm{kDa}$ on small proteins such as the Sm proteins may interfere with the ability of the protein to properly assemble into snRNP particles. To further study the assembly of the tagged proteins into snRNP, the fractionation of snRNPs and the tagged proteins were examined by an FPLC Superdex-200 column. The protein was subjected to Western analysis, and the snRNAs were analyzed by primer extension. The results presented in Figure $3 \mathrm{~B}$ demonstrate that fractionations 18-22 carry the majority of SL and U2 snRNPs. The U4 was found in fractions $12-20$, U5 in two peak fractions

FIGURE 2. Affinity selection of snRNAs using TAP-tagged Sm proteins. Whole cell extracts were prepared from induced cells $\left(500 \mathrm{~mL}, 10^{7}\right.$ cells $\left./ \mathrm{mL}\right)$ carrying the TAP-tagged Sm proteins, as described in Materials and Methods. (A) The extracts were subjected to Western analysis using IgG antibodies. The level of the RNA binding protein KH-4A was used to control for equal loading (antibodies were diluted 1:5000). (B- $a$ ) Primer extension to detect the level of selected snRNAs. Extracts were subjected to affinity selection using IgG agarose beads. RNA was prepared from an aliquot of cells before selection (Total, designated T), after affinity selection from the supernatant (designated S), and RNA from the beads (designated B). From the total RNA and the supernatant only an aliquot (2\%) of the sample was analyzed, whereas the entire sample from the beads was analyzed. The RNA from these samples was analyzed by primer extension, as described in Figure 1A. (B-b) Quantitative analysis to evaluate the specific selection of snRNAs was performed on data obtained from three independent experiments. The efficiency of selection is expressed in arbitrary units, and these do not represent the exact percentage of selection from the entire population of snRNPs. $(C)$ Localization of the SmE-GFP. Cells were fixed and subjected to in situ hybridization as described in Materials and Methods. Fluorescence of GFP and anti-DIG rodamine was examined. Cells expressing the SmE-GFP proteins were hybridized with DIG-labeled SL RNA probe. (C-a) FISH with SL RNA. (C- $b$ ) Fluorescence of SmE-GFP. (C-c) Merged images of $a$ and $b$. $(C-d)$ The nuclei stained with DAPI merged with images of $a$ and $b$. Bar, $1 \mu$ m. 
A

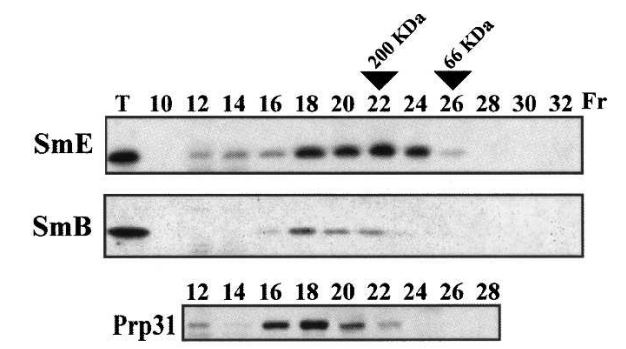

B

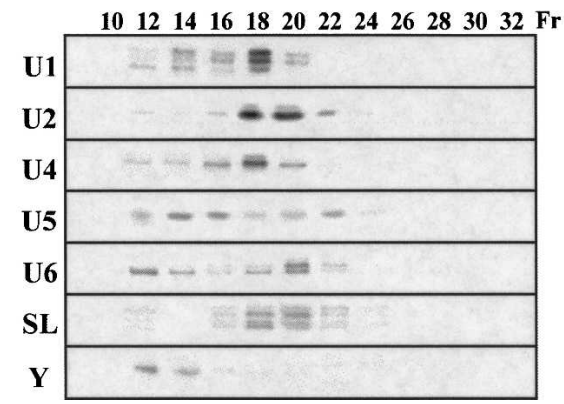

FIGURE 3. Fractionation of extracts from cells expressing TAPtagged Sm proteins and the Prp31-BB2 tagged protein on FPLC. Whole cell extracts were prepared from cells $\left(500 \mathrm{~mL}, 10^{7}\right.$ cells $\left./ \mathrm{mL}\right)$ expressing the fusions as described in Materials and Methods. (A) Proteins were subjected to Western analysis using IgG or anti-BB2 antibodies. (T) Designates $2 \%$ of total extract loaded on the column. Twenty percent of each fraction was analyzed. The identity of the protein examined is indicated. The elution positions of marker proteins BSA $(66 \mathrm{kDa})$ and $\beta$-amylase $(200 \mathrm{kDa})$ are indicated with arrows. (B) Primer extension to analyze the fractionation of snRNPs. RNA was extracted from every other fraction and analyzed by primer extension for the levels of U1, U2, U4, U5, U6 SL RNA the Y structure intermediate.

(14-16 and 20-22), whereas the U6 was also found in two peak fractions (12-14 and 18-22). The two peaks observed for U5 and U6 snRNA suggest that these RNAs are present in monoparticles as well as in multimeric particles. Indeed, dimeric U4/U6 and trimeric U4/U5/U6 were recently observed in trypanosomes by sucrose gradient fractionation (Liang et al. 2006), and these particles most probably are found in fractions 14-16 carrying U4/U5/U6 snRNAs.

The tagged SmE protein that is common to all snRNPs was found in fractions 12-24, which are enriched in all the snRNPs and SL RNP (Fig. 3A). No signal was observed in fractions 32 and above, where free protein should fractionate, suggesting that all the tagged cellular proteins are bound to snRNAs. Sm proteins and all snRNAs including SL RNA and the Y-structure intermediate were also found in fractions 12-14 (Fig. 3A,B). These fractions contain large spliceosomal complexes carrying also splicing factors such as SF1 and U2AF65 (D. Mualem, M.Z. Stern, and S. Michaeli, unpubl.). When compared to the distribution of SmE, the tagged SmB protein, which binds only to U1, U4, U5, and SL RNA, was less broadly distributed (Fig. 3A).

The fractionation of the tagged $\mathrm{Sm}$ proteins resembles the fractionation of a tagged Prp31 protein (Fig. 3A). This protein is associated with U4 and the tri-snRNP complex (Liang et al. 2006). The Prp31 was tagged at the $\mathrm{N}$ terminus with a BB2 epitope. The second allele was disrupted, and therefore all the cellular Prp31 is epitope-tagged (Liang et al. 2006). The fractionation of Sm- and Prp31-tagged proteins suggest that based on these examples, regardless of the tagging procedure, abundant RNA binding proteins such as Sm core proteins or particle-specific proteins (Prp31) are assembled efficiently into spliceosomal complexes. We cannot rule out the possibility that tagged proteins that did not assemble with snRNAs were subjected to proteolysis.

Our results also do not show correlation between the level of expression of the tagged protein and the efficiency of selection of snRNPs. For instance, SmD2 is highly expressed compared to other Sm proteins, yet it selects the snRNAs as well as SmG, which is expressed at lower levels (compared to SmD2). All these data suggest that the differential efficacy of affinity selection may reflect the ability of the IgG to bind to the protein A domain within the tagged protein when the protein is assembled in the snRNP complex.

\section{Bioinformatics searches identified three novel $\mathrm{Sm}$-like proteins}

The differential binding of U2 and U4 to core Sm proteins prompted us to search for additional proteins harboring Sm motifs. We previously have described seven proteins in the T. brucei genome that contain Sm motifs. Silencing two of these proteins, Lsm3 and Lsm8, results in destabilization of U6 snRNA and mRNA. Therefore, these proteins are bona fide Lsm proteins (Liu et al. 2004). Bioinformatics analysis was expanded, and three data sets were prepared for trypanosome proteins carrying Sm motifs. These included the Sm core proteins (Palfi et al. 2000), Lsm proteins (Liu et al. 2004), and three proteins that we termed SSm (see below). The bioinformatics analysis was performed using the program s-search from the GCG package (version 11.1; Accelrys Inc.), which conducts a rigorous Smith-Waterman search for similarity between a query sequence and a group of sequences of the same type. The purpose of these searches was to identify the relatedness of the SSm proteins to known Sm or Lsm proteins. The protein SSm2-1 (Tb927.6.4340) was previously designated Lsm5 (analogous to SmE) (Liu et al. 2004). When the SSm2-1 was compared to all the Sm core proteins, it was clear that this protein was related to the following proteins: $\mathrm{SmF}$ with the highest score, then SmD2 and SmB. However, when SmB was the query against the SSm proteins, the SSm2-1 protein was selected with the highest score. We next performed pairwise alignments between SSm2-1 and each of the Sm proteins specified above. The results indicate that when SSm2-1 is compared with SmF, the second 
Sm motif deviates strongly from the defined SmF motif (Hermann et al. 1995). The same analysis was performed with SmD2, and showed that the first Sm motif deviates from the consensus $S \mathrm{~m}$ motif 1 of this protein. However, SSm2-1 resembles SmB in both the sequence and the length of the two Sm motifs. The relatedness between SSm2-1 and $\mathrm{SmB}$ is presented in Figure 4A. The protein shares 25\% identity and $33 \%$ similarity with the human SmB protein. The same analysis was performed on SSm2-2, a protein carrying an Sm motif identified in this study (Tb10.70.2250). The results indicate that this protein is most closely related to SmD3. Searching the Sm data set against the SSm proteins identified SSm2-2 as the one most closely related to SmD3. The multiple alignment of SSm2-2 with $\mathrm{SmD} 3$ is presented in Figure 4B. Indeed, the protein shares 33\% identity and 52\% similarity with human SmD3. Performing the same analysis on SSm4 (Tb927.7.6380), which was previously annotated as Lsm2 (Liu et al. 2004), indicated that this protein is related to $\mathrm{SmF}, \mathrm{SmG}, \mathrm{SmE}$, and $\mathrm{SmB}$ with decreasing scores. The highest similarity was found to SmF. However, the distance between the Sm motifs of this protein and that of SmF deviates considerably. Based on these bioinformatics considerations, it is impossible to assign to which of the Sm proteins SSm4 is most closely related. However, one unique characteristic of $\mathrm{SmD} 3$ is an E (glutamic acid) at the fourth position of $\mathrm{Sm}$ motif 1 . This unique property exists in SSm4 as well as in SSm2-2 and Lsm4 (homologous to SmD3) (Liu et al. 2004). These data suggest that SSm4 is related to SmD3 protein. The alignment of $\mathrm{SSm} 4$ with $\mathrm{SmD} 3$ is presented in Figure 4C.

\section{Silencing by RNAi and TAP-tagging of Sm-like proteins reveal novel proteins that bind specifically to U2 and U4 snRNAs}

To examine whether the additional Sm-motif-containing proteins are part of snRNA complexes, SSm2-1, 2-2, and 4 were independently knocked down by RNAi using the T7opposing system. Transfected cells were induced for dsRNA production for $3 \mathrm{~d}$ and then analyzed for U snRNA levels by primer extension. In SSm2-1- and SSm2-2-depleted cells, the level of U2 was specifically reduced. In SSm4depleted cells, the level of U4 snRNA was significantly reduced. In no case did silencing of an SSm protein result in U6 snRNA destablization (Fig. 5A-a). The quantitation of reduction in $\mathrm{U}$ snRNA level (based on three independent experiments) is presented in Figure 5A-b. The SL RNA level in cells silenced for the SSm proteins increased by twofold. The effect on SL RNA in SSm-silenced cells resembles the effect we previously observed during knockdown of Lsm3 and Lsm8. In these cells, the level of SL RNA increased by approximately twofold (Liu et al. 2004). The increase in SL RNA during Lsm and SSm silencing is most probably a

A

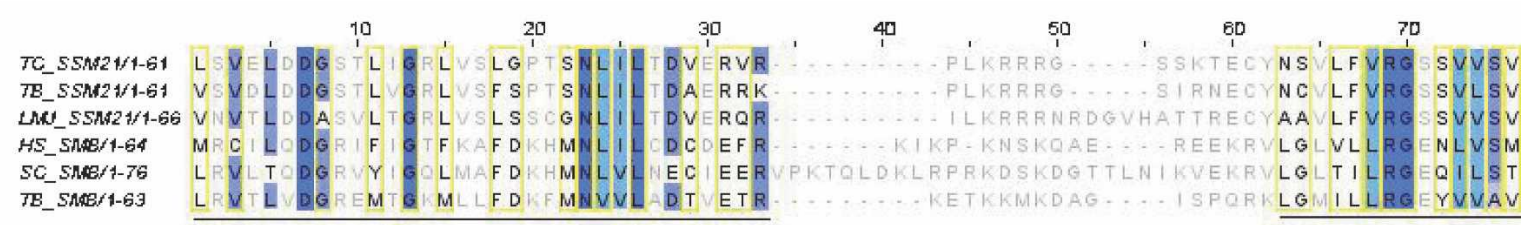

B

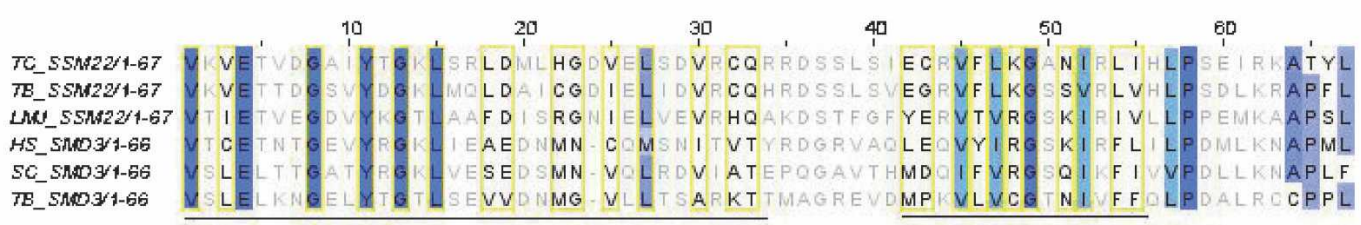

C

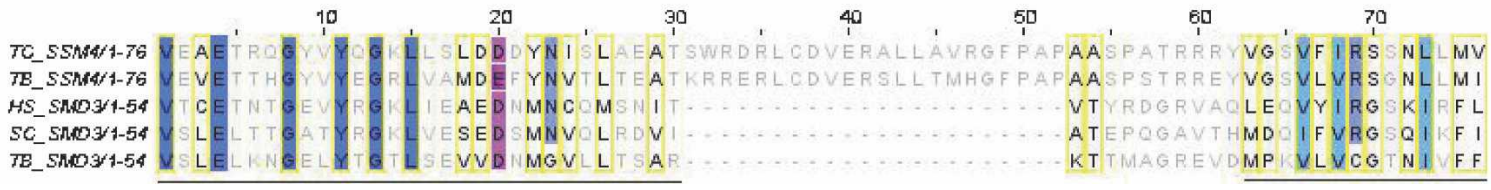

FIGURE 4. An alignment of SSm with related Sm proteins. (A) Alignment of SSm2-1 with SmB. (B) Alignment of SSm2-2 with SmD3. (C) Alignment of SSm-4 with SmD3. The alignment was performed using the CLUSTALW program and presented by the Jalview program (Clamp et al. 2004). Conserved amino acids are highlighted as follows: highly conserved amino acids in dark blue, aliphatic amino acids (I,V,L) in turquoise, conserved amino acids in light purple, and negatively charged amino acids (D,E) in pink. The Sm motifs defined by Hermann et al. (1995) are underlined, and amino acids that constitute the core motif are boxed in yellow. (HS) Homo sapiens, (SC) Saccharomyces cerevisiae, (TB) T. brucei, (TC) Trypanosoma cruzi, (LMJ) Leishmania major. The sequences were obtained from the following accession numbers: The human and the yeast SmD3 and SmB are AAA57034, P14678, AAB32821, and P40018, respectively. The T. brucei SmD3, SmB, SSm2-1, SSm2-2, and SSm4 are Tb927.4.890, Tb927.2.4540, Tb927.6.4340, Tb10.70.2250, and Tb927.7.6380, respectively. The T. cruzi and Leishmania SSm2-1, Sm2-2, and SSm4 are Tc00.1047053506943.114, Tc00.10470535065883.10, Tc00.1047053511323.20, LmjF30.3015, LmjF36.0535, and LmjF17.0300, respectively. 
A

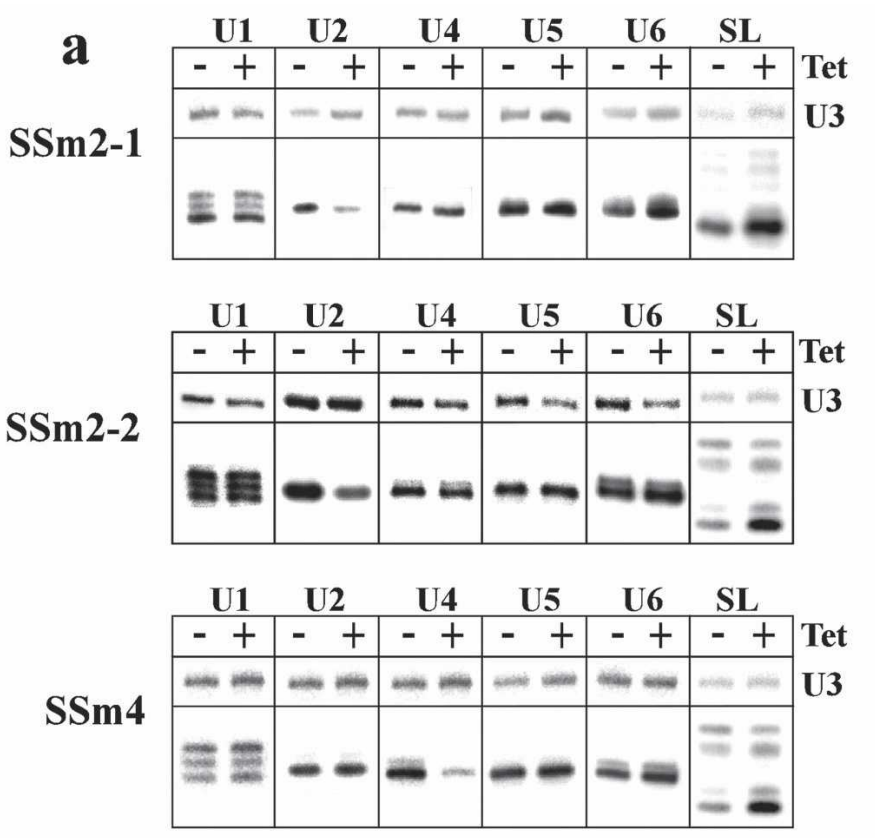

B

C
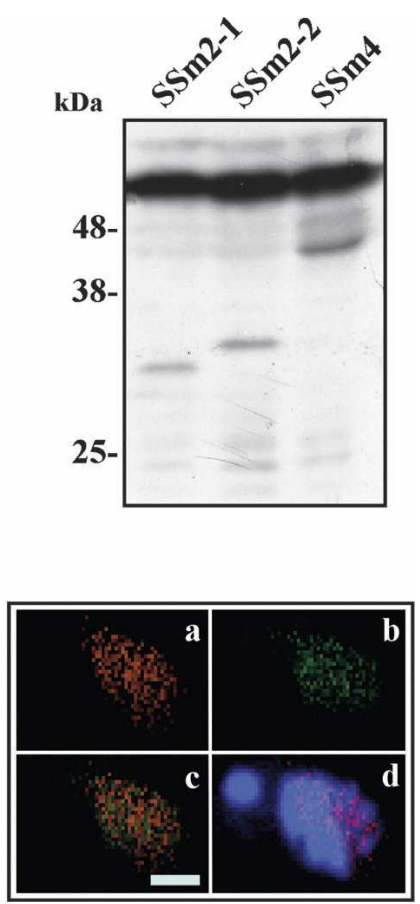

b
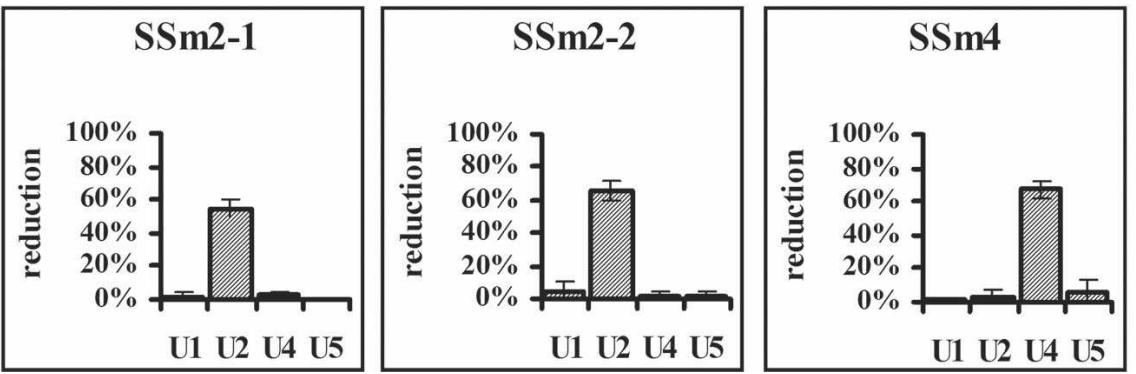

FIGURE 5. Effect of silencing of SSm proteins on the level of snRNAs and SL RNA. (A-a) Total RNA was prepared from cells carrying the SSm21, SSm2-2, and SSm4 RNAi constructs before (-Tet) and after $3 \mathrm{~d}$ of induction with tetracycline (+Tet). Total RNA (10 $\mu \mathrm{g})$ was subjected to primer extension, as described in Figure 1A. $(A-b)$ Quantitative analysis was performed as in Figure 1B, from data obtained from three independent experiments. $(B)$ Western analysis was performed to monitor the expression of SSm-TAP tagged proteins. Extracts were prepared from the cells after tetracycline induction with $50 \mathrm{ng} / \mathrm{mL}$, and were subjected to Western analysis with IgG. The level of $4 \mathrm{~A}-\mathrm{KH}$ was used to control for the amount of protein in each lane. (C) Localization of SSm2-1. Cells were fixed and subjected to in situ hybridization, as described in Materials and Methods. The fluorescence of GFP and anti-DIG rodamine was examined. Cells expressing the SSm2-1-GFP protein were hybridized with DIG-labeled U2 RNA probe. ( $C-a)$ FISH with U2 snRNA. (C-b) Fluorescence of SSm2-1-GFP. (C-c) Merged images of $a$ and $b$. $(C-d)$ The nuclei stained with DAPI merged with images of $a$ and $b$. Bar, $1 \mu \mathrm{m}$.

secondary effect, and stems from the inhibition in transsplicing due to the reduction in the level of the spliceosomal U snRNAs (Liu et al. 2004).

To further elucidate the precise interactions of these three SSm proteins with specific RNAs, TAP-tagged proteins were generated for SSm2-1, 2-2, and SSm4. The expression of the tagged proteins was verified by Western analysis (Fig. 5B). We next examined the localization of the GFP-fused SSm 2-1. The images presented in Figure 5, C-a and C-b, suggest that both the U2 snRNA and the GFPfused protein are found in speckles. Colocalization of the U2 snRNA and the fused SSm protein is demonstrated in the merged pictures (Fig. 5C-c,C-d). As opposed to the
SmE that was confined to a large dot and numerous speckles, the SSm was found only in speckles.

To examine whether the TAP-tagged proteins are associated with the cognate snRNAs, extracts were fractionated in an FPLC Superdex-200 column, and the distribution of the tagged proteins was examined by Western analysis. The localization of the particles was examined by primer extension with U2 and U4 snRNA probes (Fig. 6A-a,A-b). The results demonstrate the cofractionation of the tagged proteins with the cognate RNAs. No free tagged proteins were observed.

After verifying that the tagged proteins are properly assembled into particles, the association of the tagged 
A

$\boldsymbol{a}$

SSm2-2

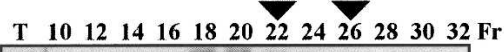

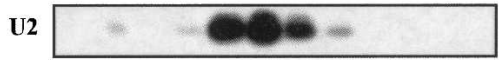

b

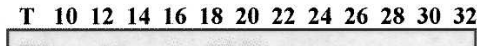

SSm4

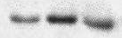

U4

$-\cdots$

B

a

U3

SSm2-1

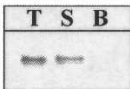

b

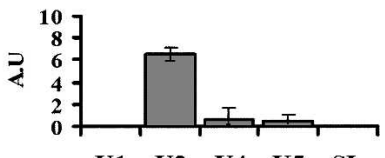

U1 U2 U4 U5 SL
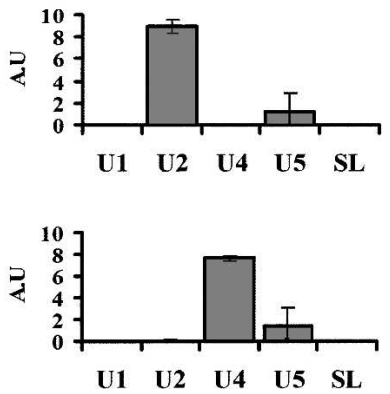

FIGURE 6. Fractionation of extracts from TAP-tagged SSm proteins on FPLC. (A) Whole cell extracts were prepared from cells $(500 \mathrm{~mL}$, $10^{7}$ cells $/ \mathrm{mL}$ ) expressing the SSm fusions as described in Materials and Methods. (A-a) Western analysis of SSm2-2 and its colocalization with U2 snRNA. Proteins were subjected to Western analysis using IgG antibodies. (T) Designates $2 \%$ of total extract loaded on the column. Twenty percent of each fraction was analyzed. The identity of the protein examined is indicated. The same fractions were deproteinized, and the RNA was subjected to primer extension with U2 antisense oligonucleotide. ( $A-b)$ Western analysis of SSm4 and its colocalization with U4 snRNA. The same as in $A$ - $a$ but using oligonucleotide complementary to U4 snRNA. The elution position of marker proteins BSA (66 kDa) and $\beta$-amylase $(200 \mathrm{kDa})$ are indicated with arrows. (B-a) Specific affinity selection of snRNAs by SSm proteins. Whole cell extracts were prepared from induced cells $\left(500 \mathrm{~mL}, 10^{7}\right.$ cells $\left./ \mathrm{mL}\right)$ carrying the SSm2-1, SSm2-2, and SSm4, TAP-tagged constructs as described in Materials and Methods. The extracts were subjected to affinity selection using IgG agarose beads. RNA was prepared from an aliquot of cells before selection (Total, designated T), after affinity selection from the supernatant (designated S), and RNA from the beads (designated B). From the total RNA and the supernatant only an aliquot of the sample was analyzed (SSm2-1, 1/70; SSm2-2, 1/50; SSm4, 1/60), whereas the entire sample from the beads was analyzed. The RNA from these samples was analyzed by primer extension, which was performed as described in Figure 1A. $(B-b)$ Quantitative analysis to evaluate the specific selection of snRNAs. The analysis was performed on data from three independent experiments. The efficiency of selection is given in arbitrary units.

protein with snRNA was examined after affinity selection by primer extension. The results are presented in Figure $6 \mathrm{~B}-\mathrm{a}$ and quantified in Figure 6B-b (based on three independent experiments). SSm2-1 was selectively associated with U2 snRNA; only trace amounts of the other U snRNAs were bound to the protein preparations. $\mathrm{SSm} 4$ is uniquely associated with U4 snRNA. Cofractionation of U4 with U5 was also observed; this is most likely a result of the presence of tri-snRNP complexes (Liang et al. 2006). The affinity selection of the U4 with U5 in this experiment suggests that the affinity purification methodology is more efficient in retaining the tri-snRNP complex than are the sucrose- gradient-based procedures (Liang et al. 2006). SL RNA was not selected with TAP-tagged SSm2-1, SSm2-2, and SSm-4. The data suggest that $\mathrm{SSm}$ proteins selectively associate with a specific subset of $U$ snRNAs.

\section{DISCUSSION}

In this study, we have characterized the function of $10 \mathrm{Sm}$ and Sm-related proteins that are important for snRNP assembly in trypanosomes. Seven of these proteins, Sm B, D1, D2, D3, E, F, and G, were previously believed to constitute the heptameric core $\mathrm{Sm}$ proteins that are known to 
associate with all $\mathrm{U}$ snRNAs in other eukaryotic cells. We have demonstrated that different sets of Sm proteins bind to different $U$ snRNAs to form specific snRNPs. Our intracellular localization studies reveal that in trypanosomes, Sm proteins are present in the nucleus of parasites, possibly in specific subnuclear locales. Three newly identified proteins carrying Sm motifs, SSm2-1, 2-2, and 4, unique to trypanosomes, are part of the core Sm assembly on specific snRNAs. These proteins localize to nuclear speckles that are distinct from the nuclear compartment where SL RNA Sm assembly most probably takes place.

In yeast and mammals, the same complement of core Sm proteins binds $\mathrm{U} 1, \mathrm{U} 2, \mathrm{U} 4$, and $\mathrm{U} 5$ to the snRNP complex necessary for spliceosome function (Will and Lührmann 2001). Mammalian Sm proteins form three subcomplexes, namely, D1/D2, D3/B, and E/F/G, in in vitro reconstitution experiments (Hermann et al. 1995; Raker et al. 1996). Two additional core Sm proteins exist in mammals; one is an isoform of SmB (van Dam et al. 1989), and another an isoform of the same protein that localizes exclusively to neurons and is known as $\mathrm{N}$ protein (Schmauss et al. 1989). These proteins most probably also assemble into snRNP complexes in the cytoplasm, but it is currently unknown whether particles carrying these special Sm proteins have unique functions in regulated or constitutive splicing.

Results suggest that different snRNPs in T. brucei assemble using different subsets of core Sm proteins. This was observed most strikingly in the case of the $\mathrm{U} 2$ and $\mathrm{U} 4$ snRNPs. In the U2 snRNP, the $\mathrm{SmB} / \mathrm{D} 3$ protein complex is replaced by the SSm2-1 and SSm2-2 proteins. It was not surprising to find a pairwise swap, since $\mathrm{SmB} / \mathrm{D} 3$ normally associate into a subcomplex during $\mathrm{Sm}$ assembly (Raker et al. 1996). However, this does not seem to be the case for the U4 snRNP, as $\mathrm{SmB}$ is retained in the U4 snRNP, but $\mathrm{SmD} 3$ is replaced by the unique SSm4 protein. SmB associates with U4 snRNP but not with U2 snRNP, indicating that an $\mathrm{SmB}$ can function independently from SmD3. This conclusion is based on the following results: (1) silencing of SmD3 reduced the level of U4; (2) tagged SmD3 did not select U4; (3) SmB silencing reduced the level of U4 snRNA; and (4) tagged SmB binds U4 snRNA. Taken together, these data suggest that trypanosome $\mathrm{SmB}$ may have more than one partner. Surprisingly, the trypanosome SmB does not deviate substantially from its counterpartners in other eukaryotes (Palfi et al. 2000) to explain its promiscuity in interactions with different Sm and SSm proteins.

Although the results regarding the effect on snRNAs in RNAi-silenced cells and the differential binding of the TAPtagged proteins to snRNAs are consistent with a unique core composition of the $\mathrm{U} 2$ and $\mathrm{U} 4$ snRNPs, massspectrometry analysis of pure core Sm heptameric complexes is necessary. Such analysis is needed, as the efficiency of IgG-based capture of Sm complexes containing different $\mathrm{U}$ snRNAs varied. This may stem from the different availability of the tagged protein to bind to the IgG-containing resin, as binding likely is affected by steric hindrance by the three-dimensional configuration of the RNA-protein complex.

What is the precise function of SSm proteins in trypanosomes? Two striking unique properties of trypanosome U snRNAs, compared to these RNAs in other eukaryotes, may explain the requirement for these extra proteins. First, snRNAs that participate in splicing possess different caps; the SL RNA has a special cap-4 structure; U1, U2, and U4 snRNAs possess a trimethylguanosine cap; and U5 does not have a cap while U6, as in other eukaryotes, possesses a $\gamma$-methyl cap. Second, as opposed to eukaryotic snRNAs (U1, U2, U4, and U snRNAs) whose Sm-sites comply with the Sm consensus $\mathrm{PuAU}_{4-6} \mathrm{GPu}$ (Liautard et al. 1982), several of the trypanosome $\mathrm{Sm}$-sites are degenerate and deviate strongly from this consensus. The T. brucei Sm binding site of the SL RNA deviates slightly (AAUCUGG) from the canonical Sm-site, because of the C substitution in the U-rich region (Bruzik et al. 1988). Also, the T. brucei U5 Sm-recognition site differs from the consensus and is AACUUUG, also carrying the C substitution (Dungan et al. 1996; Xu et al. 1997). In contrast, the Sm-site deviations found in trypanosome U2 and U4 snRNAs (Mottram et al. 1989) involve substitution of pyrimidine with purines. Specifically, the T. brucei $\mathrm{U} 2$ and U4 sites are AACUGUUG and AAGUUG, respectively. Recognition of these degenerate Sm-sites likely requires SSm proteins. Indeed, U2 and $\mathrm{U} 4$, which have the most degenerate Sm-sites, bind the unique SSm proteins. These findings are reminiscent of the finding about the mammalian U7 snRNP. In U7 snRNP, $\mathrm{SmD} 1$ and $\mathrm{SmD} 2$ are replaced by two unique Sm-like proteins, Lsm10 and Lsm11. Interestingly, Lsm10 and 11 binding requires the degenerate $\mathrm{Sm}$-site found in the U7 snRNA; replacing this site with a consensus Sm-site blocks Lsm10 and 11 binding to U7 snRNA. Whereas in the case of mammalian U7 the deviations of the Sm-site are in the $3^{\prime}$ half of the Sm-site, in trypanosomes the changes are more extensive and also exist in the $5^{\prime}$ half of the Sm-site, thus suggesting that the differential binding of $\mathrm{Sm} / \mathrm{SSm}$ might be more complex than in the case of the U7 snRNP. Experiments are in progress to determine whether the degenerate Sm-site is the only factor that helps discriminate $\mathrm{Sm}$ from SSm binding by performing in vitro binding and reconstitution of U2 snRNP harboring a canonical Sm-site, and of SL RNA carrying the degenerate U2 snRNA site. Most recently it was found that Lsm 10 and 11 do not undergo dimethylarginine modification. This may explain in part the specificity of these proteins to the degenerate Sm-site (Azzouz et al. 2005). In trypanosomes, the specific interactions of the SSm over Sm core proteins cannot be attributed to dimethyl modification, since most of the trypanosome Sm proteins lack the RG repeats required for these modifications.

Our studies suggest that an SL RNA Sm-specific protein may not exist, since seven Sm core proteins were shown to 
bind SL RNA in this study. The subcellular site of SL RNA assembly with Sm proteins is currently subject to debate. It was initially proposed that since cap-4 formation is cotranscriptional, SL RNA assembly takes place in the nucleus (Mair et al. 2000). Later it was suggested that XPO1, an export factor, may mediate the transport of the SL RNA from the nucleus to the cytoplasm, suggesting that at least part of the assembly process may occur in the cytoplasm (Zeiner et al. 2003). The observation of large cytoplasmic SL RNA speckles (SL RNP-C) during Sm depletion supports the cytoplasmic assembly of SL RNA. The fact that the SL RNA in SL RNP-C lacks the +4 cap modification is also reminiscent of the linkage that exists in mammals between the formation of the trimethylguanosine cap and cytoplasmic Sm assembly (Mandelboim et al. 2003). However, recent data from our laboratory suggest that several hours following depletion of Sm proteins, defective SL RNA lacking the +4 cap modification accumulates in the nucleus, suggesting that $\mathrm{Sm}$ assembly takes place in this compartment (Biton et al. 2006). Furthermore, the methyltransferase that directs modification on SL RNA at the +3 and +4 positions, as well as the enzyme that carries the modification at +2 position, were recently identified and are both nuclear proteins (Arhin et al. 2006a,b). All these recent data strongly suggest that SL RNA assembly with Sm proteins takes place in the nucleus. The distinct nuclear "dot" carrying the SmE-GFP fused protein that colocalizes with the SL RNA most probably is the site where SL RNP is produced. These data agree with the recent observation that SL RNA transcription takes place in a distinct nuclear structure that appears as "dot-like" under confocal microscopy (Dossin and Schenkman 2005). Interestingly, the localization of the SSm2-1 protein in the nucleus suggests that U2 snRNP does not exist in the SL RNA factory, but rather in small speckles, similar to the localization of splicing-factor speckles recently observed in T. brucei (Liang et al. 2006). "Speckles" in metazoa are dynamic structures in which both the protein and RNA components cycle continuously to other nuclear locations. They are a reservoir for splicing factors and snRNPs that are recruited to the site of transcription/ splicing of pre-mRNA (Lamond and Spector 2003). The unique compartment carrying SL RNA observed in this study may be the site of production as well as storage for SL RNP.

In summary, this study identifies the core Sm proteins that bind the SL RNA and the SSm proteins that bind specifically to $\mathrm{U} 2$ and $\mathrm{U} 4$ snRNAs. SSm proteins may assist trypanosomes in sorting the different snRNAs to acquire different cap structures, and to correctly and efficiently assemble $\mathrm{Sm}$ proteins despite having degenerate Sm sites. Localization of SSm and Sm proteins suggests that the majority of SL RNA is found in the cell in a special nuclear compartment that might be the site for SL RNP production.

\section{MATERIALS AND METHODS}

\section{Oligonucleotides}

3977: 5-CGCTTTCGCTTCCCAC, antisense, complementary to positions 54-68 of U1 snRNA.

3974: 5-GAACAGTTTAATAAC, antisense, complementary to positions $31-45$ of U2 snRNA.

3976: 5-TGCCGTTCATCGAAC, antisense, complementary to positions 107-121 of U3 snRNA.

2593: 5-CAAACTTTCCCCGAAGGA, antisense, complementary to positions $76-93$ of U4 snRNA.

3975: 5-CCGCTCGAGGACACCCCAAACTTT, antisense, complementary to positions $48-72$ of U5 snRNA.

19282: 5-AGCTATATCTCTCGAA, antisense, complementary to positions $81-95$ of U6 snRNA.

1098: 5-GGGAGCTTCTCATAC, antisense, complementary to positions $40-54$ of SL snRNA.

P013B10: 5'-AACTAACGCTATTATTA, sense, complementary to positions 1-17 of SL RNA used for PCR amplification of the 161-bp fragment for in situ hybridization.

0244C02: 5' -TTAATACGACTCACTATAGGGAGAAAAAAAATA AAAAAAATA, antisense, complementary to positions 143-161 of SL RNA used for PCR amplification of the 161-bp fragment for in situ hybridization.

11,240,946 TBU2-5S: 5'-ATATCTTCTCGGCTATTTAG, sense, complementary to positions $112-131$ of U2 snRNA used for PCR amplification of the 148-bp fragment for in situ hybridization.

9001890: 5'-ACCGTCGCGCTCCGTCCGGA, antisense, complementary to positions 240-259 of U2 snRNA used for PCR amplification of the 148-bp fragment for in situ hybridization.

21113: 5'-AACTAACGCTATTATTA, sense, complementary to positions $1-17$ of SL RNA.

9091: 5'-GCAGGAACCAACAGCACAATGCG, antisense, complementary to positions $90-110$ of SL RNA.

11240946: 5'-ATATCTTCTCGGCTATTTAG, sense, complementary to positions $1-20$ of U2 snRNA.

11643974: 5'-GAACAGTTTAATAAC, antisense, complementary to positions $31-45$ of U2 snRNA.

\section{Plasmids}

For RNAi silencing of the Sm and SSm proteins, PCR fragments, generated from the following oligonucleotides, were inserted into the pZJM vector:

SmB; 7414: 5' -CCGCTCGAGATGCTTCACAACATCAACCG, sense, from position 16-35 including an XhoI site.

7415: 5'-CCCAAGCTTTCAAAGTTTGCTGGTTGTGC, antisense, complementary to positions 262-280 including a HindIII site.

SmD2; 0943: 5' -CCGCTCGAGGAGGAACCCACTAAATTGCAGC, sense, from positions 10-31 including an XhoI site.

0944: 5' -CCCAAGCTTTCCCGCAACACGAGGTTAAAG, antisense, complementary to positions 201-220 including a HindIII site.

SmD3; 1036: 5'-CCGCTCGAGAAGGTGCTGTCCGATGCTG, sense, from positions 25-43 including an XhoI site.

1037: 5'-CCCAAGCTTCACCAAATCCCTTTCCGTC, antisense, complementary to positions 298-316 including a HindIII site.

SmF; 7416: 5' -CCGCTCGAGATGGATGCAAATGTACCG, sense, from positions $1-18$ including an XhoI site. 
7417: 5'-CCCAAGCTTTGGTACTTCCCGAATGTACAAC, antisense, complementary to positions 198-219, including a HindIII site.

SmG; 0945: 5'-CCGCTCGAGTGAACCACTTCATGGAGAAGC, sense, from positions 29-49 including an XhoI site.

0946: 5'-CCCAAGCTTTATCTTCGTTCTCGACAGTACCC, antisense, complementary to positions 159-180 including a HindIII site.

SSm2-1; 13777408: 5' -CCGCTCGAGGTGTGGATTTGGATGACG, sense, from positions 56-73 including an XhoI site.

13777409: 5' -CCCAAGCTTTGTGTCCAAACTTTGACTCG, antisense, complementary to positions 323-341 including a HindIII site.

SSm2-2; 0633: 5'-CCGCTCGAGTCCCTTACGTGCCATTTGTG, sense, from positions $21-40$ including an XhoI site.

0633: 5'-CCCAAGCTTAGACAAATTCTTCCAGTCCAGG, antisense, complementary to positions 255-276 including a HindIII site.

SSm4; 13238819: 5'-CCGCTCGAGTCGTGTTGTCTCTGCACCA CTG, sense, from positions 162-183 including an XhoI site.

13238820: 5' -CCCAAGCTTCTCCATGCGATTCTTCTGCC, antisense, complementary to positions 508-527 including a HindIII site.

For TAP-tagging the Sm and SSm proteins, PCR fragments, generated from the following oligonucleotides, were inserted into the pLew79-MHTAP vector:

SmB-TAP; 4771: 5'-CCCAAGCTTATGGGCCACC AAAATATGCT, sense, from positions 1-20 including a HindIII site.

4772: 5'-CCCGGATCCATCGCGTTTCCGCTTGG, antisense, complementary to positions 310-330 including a BamHI site.

SmD1-TAP; 1165: 5'-CCCAAGCTTATGCCCGCGGCGGAGT, sense, from positions 1-20 including a HindIII site.

1164: 5'-CCCGGATCCCTCATTTGATCGCTCCGTCC, antisense, complementary to positions 298-318 including a BamHI site.

SmD2-TAP; 1707: 5'-CCCAAGCTTATGTCTGGTGAGGAACCC ACT, sense, from positions 1-21 including a HindIII site.

1849: 5'-GCCGCAGTGGGATCCTACAGCGCTCTGCGGCAA, antisense, complementary to positions 316-332 including a BamHI site.

SmD3-TAP; 1162: 5'-CCCAAGCTTATGAACACGGAGGGGCT, sense, from positions 1-18 including a HindIII site.

1163: 5'-CCCGGATCCCTTCTTTGGCTTCTTACGG, antisense, complementary to positions 327-345 including a BamHI site.

SmE-TAP; 9047: 5-CCCAAGCTTATGAGCGTCACAACAAAGCA, sense, from positions 1-20 including a HindIII site.

9048: 5-CGCGGATCCTATGCCAATAGGGTGAATGACAC, antisense, complementary to positions 236-258 including a BamHI site.

SmF-TAP; 1156: 5'-CCCAAGCTTATGGATGCAAATGTACCG, sense, from positions 1-20 including a HindIII site.

1157: 5' -CCCGGATCCTTCCTTTGGTACTTCCC, antisense, complementary to positions 210-225 including a BamHI site.

SmG-TAP; 1158: 5'-CCCAAGCTTATGCCCGCCAAGAAGCG, sense, from positions $1-17$ including a HindIII site.

1159: 5'-CCCGGATCCCACTTCAAGACCGACAATATCC, antisense, complementary to positions 224-246 including a BamHI site.

SSm2-1-TAP; 1768: 5'-CCCAAGCTTATGTCTGTCAAATCCGA GCG, sense, from positions 1-20 including a HindIII site.
1769: 5'-CGCGGATCCTCGGAGGGGAGTGTCCAAACTTTG, antisense, complementary to positions 323-341 including a BamHI site.

SSm2-2-TAP; 4773: 5'-CCCAAGCTTATGGAAGGTG TAGCTG ATAT, sense, from positions 1-20 including a HindIII site.

4774: 5'-CCCGGATCCTAGGAGTAGCTTTTTCTTT, antisense, complementary to positions 373-393 including a BamHI site.

SSm4-TAP; 9043: 5'-CCCAAGCTTATGTCGGTAGAGGCGAG TAG, sense, from positions 1-20 including a HindIII site.

9044: 5'-TTATGAGCTGGATCCCTCTTTCTTTGCTTTGGT, antisense, complementary to positions 613-628 including a BamHI site.

For the GFP constructs, PCR fragments coding for Sm and SSm proteins were generated from the following oligonucleotides and inserted into the puC19 vector:

Sm2-1-GFP; 4584: 5-GGGCCGCATGCATGTCTGTCAAATCCG AGCG, sense, complementary to positions 1-20 including an SphI site.

4583: 5-GGGCCTCTAGAGCTCGGAGGGGAGTGTCCAAAC, antisense, complementary to positions 332-351 including an $\mathrm{XbaI}$ site.

SmE-GFP; 4590: 5-GGGCCGCATGCATGAGCGTCACAACAAA GCAG, sense, complementary to positions 1-21 including an SphI site.

4589: 5-GGGCCTCTAGAGCTATGCCAATAGGGTGAATG, antisense, complementary to positions 240-258 including an XbaI site.

\section{Construct generation and preparation of transgenic parasites}

To obtain the silencing constructs in the T7 opposing system, the genes were amplified by PCR using the primers listed above, and the products were cloned into the pZJM vector (Wang et al. 2000). After they were linearized, the plasmids were transfected to T. brucei 29-13 cells, and transformants were selected and cloned as previously described (Mandelboim et al. 2003). To generate the TAP-tagged versions, the genes were amplified with the primers specified above. The fragments were cloned into pLew79-MHTAP (carrying also Myc-His tags between the protein and the TAP-tag, kindly provided by M. Parsons and B. Jensen, Seattle Biomedical Institute, Washington). In the case of SmD2, pLew79-TAP was used (Schnaüfer et al. 2003). After verifying the sequence, the plasmids were transfected to T. brucei 29-13 as described above. To generate the GFP-fused proteins, the gene was amplified with a forward primer carrying the SphI site (two nucleotides were added to generate the open reading frame, ORF) and a reverse primer carrying the $\mathrm{XbaI}$ site as described above. The product was cloned into a pUC19 vector carrying the GFP. The pUC19-GFP was generated by cloning the genes using SacI and BamHI sites from a BlueScript vector carrying the GFP gene that was excised from pEGFP-N1 (Clontech) using BamHI and NotI. The pUC19GFP-Sm proteins were then cloned into the T. brucei expression vector, pXS2 (Bangs et al. 1996). The plasmids were linearized with MluI, which drives their integration into the tubulin locus (Bangs et al. 1996). 


\section{TAP-tag purification of snRNPs associated with Sm and SSm proteins}

Expression of the tagged proteins was induced for $60 \mathrm{~h}$ with tetracycline $(50 \mathrm{ng} / \mathrm{mL})$. In this vector, the production of the fused protein is under the regulation of the tetracycline repressor (Schnaüfer et al. 2003). The genes encoding all Sm proteins were amplified by PCR and cloned into the TAP-expression vector. Cells $\left(300-500 \mathrm{~mL} \sim 10^{7}\right.$ cells $/ \mathrm{mL}$ ) were harvested and washed with PBS. Extracts and affinity selection with IgG-agarose beads were performed as recently described (Lustig et al. 2005). The beads were deproteinized by phenol extraction, and the released RNA was divided in five equal parts and analyzed by primer extension, as previously described (Mandelboim et al. 2003), with the snRNA-specific primers specified above.

\section{In situ hybridization and confocal microscopy of GFP-fused proteins and immunofluorescence}

PCR for preparing the digoxigeneine-11 (DIG) labeled DNA probe, was performed under standard conditions using the Expand high fidelity enzyme (Roche Molecular Biochemicals), as recently described (Mandelboim et al. 2003; Lustig et al. 2005). After fixation, hybridization, and washing as described (Lustig et al. 2005), the cells were incubated with DAPI (4'-6-diamidino-2-phenylindole) for $5 \mathrm{~min}$ to stain the nucleus. Finally, the cells were visualized with a Zeiss LSM 510 META inverted microscope.

\section{Fractionation of snRNPs by FPLC}

Extracts were prepared as previously described (Mandelboim et al. 2003). Whole cell extracts from $5 \times 10^{9}$ cells were loaded on a Superdex 200 gel filtration column (Amersham BioSciences), equilibrated with $20 \mathrm{mM}$ HEPES ( $\mathrm{pH} 7.9$ ), $10 \mathrm{mM} \mathrm{MgCl}_{2}, 150$ $\mathrm{mM} \mathrm{KCl}$, and $5 \mathrm{mM} \beta$-mercaptoethanol at a flow rate of $0.5 \mathrm{~mL} /$ min. Fractions of $0.5 \mathrm{~mL}$ were collected. RNA and protein for every other fraction (10-32) were analyzed by Western and Northern analyses. The elutions of bovine serum albumin $(66 \mathrm{kDa})$ and $\beta$-amylase $(200 \mathrm{kDa})$ were used as markers to follow the fractionation.

\section{ACKNOWLEDGMENTS}

We thank Marylin Parsons and Bryan Jensen for the TAP-Myc-His construct. We thank Vered Ozeri for her help in designing the GFP-fusions. This study was supported by a United States-IsraelBinational Science Foundation grant and by an International Research Scholars grant from the Howard Hughes Medical Institute to S.M.

Received June 1, 2006; accepted September 28, 2006.

\section{REFERENCES}

Achsel, T., Brahms, H., Kastner, B., Bachi, A., Wilm, M., and Lührmann, R. 1999. A doughnut-shaped heteromer of human Sm-like proteins binds to the $3^{\prime}$-end of U6 snRNA, thereby facilitating U4/U6 duplex formation in vitro. EMBO J. 18: 5789-5802. Arhin, G.K., Li, H., Ullu, E., and Tschudi, C. 2006a. A protein related to the vaccinia virus cap-specific methyltransferase VP39 is involved in cap 4 modification in Trypanosoma brucei. RNA 12: 53-62.

Arhin, G.K., Ullu, E., and Tschudi, C. 2006b. 2'-O-Methylation of position 2 of the trypanosome spliced leader cap 4 is mediated by a $48 \mathrm{kDa}$ protein related to vaccinia virus VP39. Mol. Biochem. Parasitol. 147: 137-139.

Azzouz, T.N., Pillai, R.S., Dapp, C., Chari, A., Meister, G., Kambach, C., Fischer, U., and Schumperli, D. 2005. Toward an assembly line for U7 snRNPs: Interactions of U7-specific Lsm proteins with PRMT5 and SMN complexes. J. Biol. Chem. 280: 34435-34440.

Bangs, J.D., Crain, P.F., Hashizume, T., McCloskey, J.A., and Boothroyd, J.C. 1992. Mass spectrometry of mRNA cap 4 from trypanosomatids reveals two novel nucleosides. J. Biol. Chem. 267: 9805-9815.

Bangs, J.D., Brouch, E.M., Ransom, D.M., and Roggy, J.L. 1996. A soluble secretory reporter system in Trypanosoma brucei. Studies on endoplasmic reticulum targeting. J. Biol. Chem. 271: 18387-18393.

Bell, M. and Bindereif, A. 1999. Cloning and mutational analysis of the Leptomonas seymouri U5 snRNA gene: Function of the Sm site in core RNP formation and nuclear localization. Nucleic Acids Res. 27: 3986-3994.

Biton, M., Mandelboim, M., Arvatz, G., and Michaeli, S. 2006. RNAi interference of XPO1 and Sm genes and their effect on the spliced leader RNA in Trypanosoma brucei. Mol. Biochem. Parasitol. 150: 132-143.

Bouveret, E., Rigaut, G., Shevchenko, A., Wilm, M., and Séraphin, B. 2000. A Sm-like protein complex that participates in mRNA degradation. EMBO J. 19: 1661-1671.

Branlant, C., Krol, A., Ebel, J.P., Lazar, E., Haendler, B., and Jacob, M. 1982. U2 RNA shares a structural domain with U1, U4, and U5 RNAs. EMBO J. 1: 1259-1265.

Bruzik, J.P., Van Doren, K., Hirsh, D., and Steitz, J.A. 1988. Trans splicing involves a novel form of small nuclear ribonucleoprotein particles. Nature 335: 559-562.

Clamp, M., Cuff, J., Searle, S.M., and Barton, G.J. 2004. The Jalview Java alignment editor. Bioinformatics 20: 426-427.

Das, A., Zhang, Q., Palenchar, J.B., Chatterjee, B., Cross, G.A., and Bellofatto, V. 2005. Trypanosomal TBP functions with the multisubunit transcription factor tSNAP to direct spliced-leader RNA gene expression. Mol. Cell. Biol. 25: 7314-7322.

Djikeng, A., Ferreira, L., D’Angelo, M., Dolezal, P., Lamb, T., Murta, S., Triggs, V., Ulbert, S., Villarino, A., Renzi, S., et al. 2001. Characterization of a candidate Trypanosoma brucei U1 small nuclear RNA gene. Mol. Biochem. Parasitol. 113: 109-115.

Dossin, F.M. and Schenkman, S. 2005. Actively transcribing RNA polymerase II concentrates on spliced leader genes in the nucleus of Trypanosoma cruzi. Eukaryot. Cell 4: 960-970.

Dungan, J.M., Watkins, K.P., and Agabian, N. 1996. Evidence for the presence of a small U5-like RNA in active trans-spliceosomes of Trypanosoma brucei. EMBO J. 15: 4016-4029.

Gilinger, G. and Bellofatto, V. 2001. Trypanosome spliced leader RNA genes contain the first identified RNA polymerase II gene promoter in these organisms. Nucleic Acids Res. 29: 1556-1564.

Goncharov, I., Palfi, Z., Bindereif, A., and Michaeli, S. 1999. Purification of the spliced leader ribonucleoprotein particle from Leptomonas collosoma revealed the existence of an Sm protein in trypanosomes-Cloning the SmE homologue. J. Biol. Chem. 274: 12217-12221.

Hermann, H., Fabrizio, P., Raker, V.A., Foulaki, K., Hornig, H., Brahms, H., and Lührmann, R. 1995. snRNP Sm proteins share two evolutionarily conserved sequence motifs which are involved in Sm protein-protein interactions. EMBO J. 14: 2076-2088.

Kambach, C., Walke, S., Young, R., Avis, J.M., de la Fortelle, E., Raker, V.A., Lührmann, R., Li, J., and Nagai, K. 1999. Crystal structures of two Sm protein complexes and their implications for the assembly of the spliceosomal snRNPs. Cell 96: 375-387.

Lamond, A.I. and Spector, D.L. 2003. Nuclear speckles: A model for nuclear organelles. Nat. Rev. Mol. Cell Biol. 4: 605-612. 
Liang, X.H., Haritan, A., Uliel, S., and Michaeli, S. 2003. trans and cis splicing in Trypanosomatids: Mechanism, factors, and regulation. Eukaryot. Cell 2: 830-840.

Liang, X.H., Liu, Q., Liu, L., Tschudi, C., and Michaeli, S. 2006. Analysis of spliceosomal complexes in Trypanosoma brucei and silencing of two splicing factors Prp31 and Prp43. Mol. Biochem. Parasitol. 145: 29-39.

Liautard, J.P., Sri-Widada, J., Brunel, C., and Jeanteur, P. 1982. Structural organization of ribonucleoproteins containing small nuclear RNAs from HeLa cells. Proteins interact closely with a similar structural domain of U1, U2, U4 and U5 small nuclear RNAs. J. Mol. Biol. 162: 623-643.

Liu, Q., Liang, X.H., Uliel, S., Belahcen, M., Unger, R., and Michaeli, S. 2004. Identification and functional characterization of Lsm proteins in Tryapnosoma brucei. J. Biol. Chem. 279: 18210-18219.

Lustig, Y., Goldshmidt, H., Uliel, S., and Michaeli, S. 2005. The Trypanosoma brucei signal recognition particle lacks the Aludomain-binding proteins: purification and functional analysis of its binding proteins by RNAi. J. Cell Sci. 118: 4551-4562.

Mair, G., Ullu, E., and Tschudi, C. 2000. Cotranscriptional cap 4 formation on the Trypanosoma brucei spliced leader RNA. J. Biol. Chem. 275: 28994-28999.

Mandelboim, M., Barth, S., Biton, M., Liang, X.H., and Michaeli, S. 2003. Silencing of Sm proteins in Trypanosoma brucei by RNA interference captured a novel cytoplasmic intermediate in spliced leader RNA biogenesis. J. Biol. Chem. 278: 51469-51478.

Mayes, A.E., Verdone, L., Legrain, P., and Beggs, J.D. 1999. Characterization of Sm-like proteins in yeast and their association with U6 snRNA. EMBO J. 18: 4321-4331.

Mottram, J., Perry, K.L., Lizardi, P.M., Lührmann, R., Agabian, N., and Nelson, R.G. 1989. Isolation and sequence of four small nuclear U RNA genes of Trypanosoma brucei subsp. brucei: Identification of the U2, U4, and U6 RNA analogs. Mol. Cell. Biol. 9: $1212-1223$.

Nakaar, V., Dare, A.O., Hong, D., Ullu, E., and Tschudi, C. 1994. Upstream tRNA genes are essential for expression of small nuclear and cytoplasmic RNA genes in trypanosomes. Mol. Cell. Biol. 14: 6736-6742.

Palfi, Z., Lucke, S., Lahm, H.W., Lane, W.S., Kruft, V., BragadoNilsson, E., Séraphin, B., and Bindereif, A. 2000. The spliceosomal snRNP core complex of Trypanosoma brucei: Cloning and functional analysis reveals seven Sm protein constituents. Proc. Natl. Acad. Sci. 97: 8967-8972.

Palfi, Z., Schimanski, B., Günzl, A., Lucke, S., and Bindereif, A. 2005. U1 small nuclear RNP from Trypanosoma brucei: A minimal U1 snRNA with unusual protein components. Nucleic Acids Res. 33: 2493-2503.

Pillai, R.S., Will, C.L., Lührmann, R., Schumperli, D., and Müller, B. 2001. Purified U7 snRNPs lack the Sm proteins D1 and D2 but contain Lsm10, a new $14 \mathrm{kDa}$ Sm D1-like protein. EMBO J. 20: 5470-5479.

Pillai, R.S., Grimmler, M., Meister, G., Will, C.L., Lührmann, R., Fischer, U., and Schumperli, D. 2003. Unique Sm core structure of U7 snRNPs: Assembly by a specialized SMN complex and the role of a new component, Lsm11, in histone RNA processing. Genes \& Dev. 17: 2321-2333.

Raker, V.A., Plessel, G., and Lührmann, R. 1996. The snRNP core assembly pathway: Identification of stable core protein heteromeric complexes and an snRNP subcore particle in vitro. EMBO J. 15: 2256-2269.

Salgado-Garrido, J., Bragado-Nilsson, E., Kandels-Lewis, S., and Séraphin, B. 1999. Sm and Sm-like proteins assemble in two related complexes of deep evolutionary origin. EMBO J. 18: 3451-3462.

Schmauss, C., McAllister, G., Ohosone, Y., Hardin, J.A., and Lerner, M.R. 1989. A comparison of snRNP-associated Smautoantigens: Human $\mathrm{N}$, rat $\mathrm{N}$ and human $\mathrm{B} / \mathrm{B}^{\prime}$. Nucleic Acids Res. 17: 1733-1743.

Schnare, M.N. and Gray, M.W. 1999. A candidate U1 small nuclear RNA for trypanosomatid protozoa. J. Biol. Chem. 274: 23691-23694.

Schnaüfer, A., Ernst, N.L., Palazzo, S.S., O'Rear, J., Salavati, R., and Stuart, K. 2003. Separate insertion and deletion subcomplexes of the Trypanosoma brucei RNA editing complex. Mol. Cell 12: 307-319.

Séraphin, B. 1995. Sm and Sm-like proteins belong to a large family: Identification of proteins of the U6 as well as the U1, U2, U4 and U5 snRNPs. EMBO J. 14: 2089-2098.

Simpson, L., Sbicego, S., and Aphasizhev, R. 2003. Uridine insertion/ deletion RNA editing in trypanosome mitochondria: A complex business. RNA 9: 265-276.

Stevens, J.R. and Gibson, W. 1999. The molecular evolution of trypanosomes. Parasitol. Today 15: 432-437.

Terns, M.P., Lund, E., and Dahlberg, J.E. 1992. 3'-End-dependent formation of U6 small nuclear ribonucleoprotein particles in Xenopus laevis oocyte nuclei. Mol. Cell. Biol. 12: 3032-3040.

van Dam, A., Winkel, I., Zijlstra-Baalbergen, J., Smeenk, R., and Cuypers, H.T. 1989. Cloned human snRNP proteins B and B' differ only in their carboxy-terminal part. EMBO J. 8: 3853-3860.

Wang, Z., Morris, J.C., Drew, M.E., and Englund, P.T. 2000. Inhibition of Trypanosoma brucei gene expression by RNA interference using an integratable vector with opposing T7 promoters. J. Biol. Chem. 275: 40174-40179.

Will, C.L. and Lührmann, R. 2001. Spliceosomal UsnRNP biogenesis, structure and function. Curr. Opin. Cell Biol. 13: 290-301.

Xu, Y., Ben Shlomo, H., and Michaeli, S. 1997. The U5 RNA of trypanosomes deviates from the canonical U5 RNA: The Leptomonas collosoma U5 RNA and its coding gene. Proc. Natl. Acad. Sci. 94: 8473-8478.

Zeiner, G.M., Sturm, N.R., and Campbell, D.A. 2003. Exportin 1 mediates nuclear export of the kinetoplastid spliced leader RNA. Eukaryot. Cell 2: 222-230. 

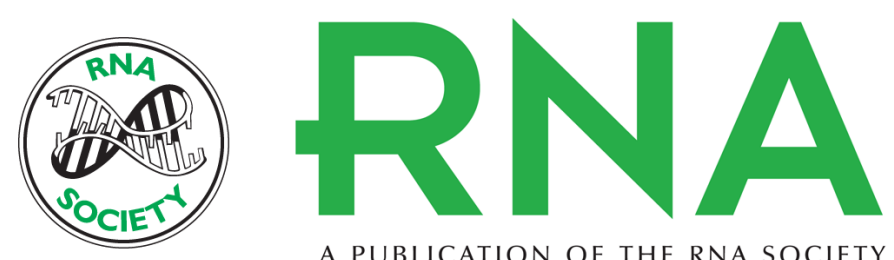

A PUBLICATION OF THE RNA SOCIETY

\section{Identification of novel snRNA-specific Sm proteins that bind selectively to U2 and U4 snRNAs in Trypanosoma brucei}

Itai Dov Tkacz, Yaniv Lustig, Michael Zeev Stern, et al.

RNA 2007 13: 30-43 originally published online November 14, 2006

Access the most recent version at doi:10.1261/rna.174307

\section{References This article cites 49 articles, 27 of which can be accessed free at: http://rnajournal.cshlp.org/content/13/1/30.full.html\#ref-list-1}

\section{License}
Email Alerting Receive free email alerts when new articles cite this article - sign up in the box at the Service top right corner of the article or click here.

To subscribe to $R N A$ go to:

http://rnajournal.cshlp.org/subscriptions 